\title{
ONLINE MONITORING AND CONTROL AQUAPONICS USING LAB
}

\section{VIEW}

\author{
Jijin C K $\mathbf{K}^{\mathbf{1}}$, Roshith $\mathrm{K}^{\mathbf{2}}$ \\ ${ }^{1} P G$ Scholar in Electrical Engineering, National Institute of Technology Calicut, Kerala, India \\ ${ }^{2}$ Assistant Professor, Department of Electronics and Instrumentation Engineering, College of Engineering Vadakara, \\ Kerala, India
}

\begin{abstract}
Aquaponics is a combination of conventional aquaculture with farming by creating an artificial ecosystem without using soil. In this system the crop are planted over a hollow tube, through which mineral and nutrition rich water is circulated with the help of an electrical pump. The excretion of fish in the tank makes the water so rich of mineral and nutrition. Plant absorbs the minerals and nutrition's from the water and the fresh water is return to the tank. This system monitors the water circulation, temperature, humidity and light inside the greenhouse and takes control action to adjust the variation from its set point. The fish feeding mechanism incorporated in the system feeds the fish daily on the basis of proper timing. The online monitor and control facility is included to take control actions by means of a web-browser from any location having internet access.
\end{abstract}

Keywords-Aquaponics; Temperature Measurement; Humidity Measurement; High Power LED Driver; Screw Conveyer; Water Detector; LabVIEW; Online Control

\section{INTRODUCTION}

At present farmers uses excessive quantity of fertilizers and pesticides by looking to the financial benefits in farming. By the continuous usage of these vegetables and fruits drastically degrades healthy body and cause serious health problems like cancer and genetic disorder for the new-born. Even though Organic farming is a solution for this disastrous situation, but the lack of fertilized land and manpower make it infeasible for a normal person. Aquaponic Farming method consumes very less space and doesn't require soil. But the tedious task of monitoring still persists. This work tries to introduce Virtual Instrumentation in Aquaponic farming by which the control action takes automatically at the same time we can monitor and adjust the control action by online.

With the help of virtual instrumentation software LabVIEW we can monitor and adjust the controller parameters of the plant from anywhere with an internet access. This project uses low cost Arduino board for interfacing the transducers with PC. A prototype is for the suggested systems is developed and tested. For large farms CCTV surveillance can be incorporated by adding cameras and interfacing cards along with software in the main server.

The first research work on growing plants without soil was reported in the book "Sylva Sylvarumby [1]" by Francis Bacon. Growing plants using mineral nutrient solutions in the abesence of soil called solution culture. Seasonal Plants can be grown irrespective of climate and climate change in controlled environment system like Aquaponics. Green Houses Management System that collect soil information with belonging environment using soil sensors, and CCTV camera has reported 2014 [2]. Mitar Simic developed system for temperature and relative humidity measurement and data acquisition using Arduino Uno and Ethernet Shield boards [3]. Thangavel Bhuvaneswari, Joshua Tan Hong Yao in 2014 tries to minimize the human care needed for the plant by automating the green house and monitor the inhouse environment status [4]. In 2015 P. S. Asolkar and Dr. U. S. Bhadade developed a system [5] that effectively monitors and controls the green house parameters of crucial importance like temperature, humidity, soil moisture, and light intensity and $\mathrm{CO} 2$ gas.

The works reported earlier has only concentrated on greenhouse and the method doesn't seems to be applied in artificical eccosystem like Aquaponics. Moreover highly sophisticated and standard control software is not used. Here we use LabVIEW which is the best available VI software so the control strategies can be improved without much change in the hardware platform.

\section{AQUAPONICS}

Aqua phonics is a food production system that combines conventional aquaculture (raising aquatic animals such as snails, fish, crayfish or prawns in tanks) with hydroponics (cultivating plants in water) in a symbiotic environment. In normal aquaculture, excretions from the animals being raised can accumulate in the water, increasing toxicity. In an aqua phonics system, water from an aquaculture system is fed to a hydroponic system where the by-products are broken down by nitrification bacteria into nitrates and nitrites, which are utilized by the plants for their growth. The cleaned water is then recirculates back to the tank. 


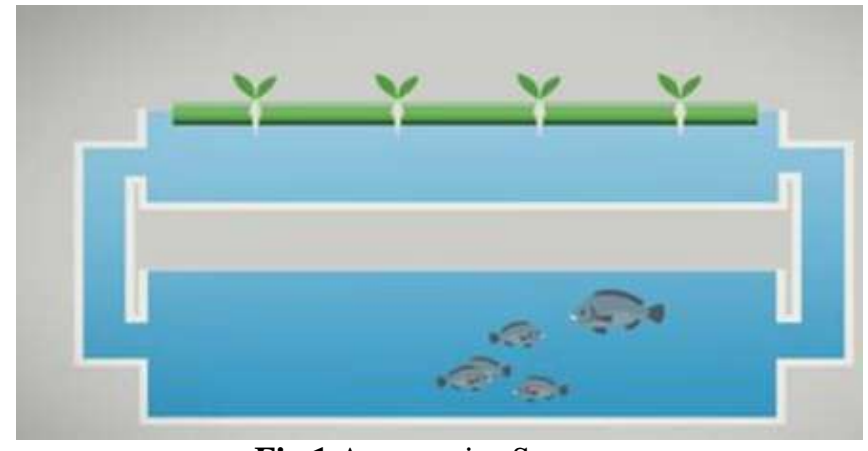

Fig 1 Aquaponics System

A commercial aquaphonics system, an electric pump moves effluent rich water from the fish tank through solids filter. Solids filter remove particles which are not decomposed and their size above that of the plants cannot absorb. The fertilized water provides nutrients for the plants and it is cleaned before returning to the fish tank, the process repeats forever in a cyclic manner. Aquaponics experiment setup can be divided in to two parts Aquaculture part and Hydroponics part. Aquaculture part mainly consists of a large tank filled with fresh water where the aquatic animals are raised. A fish feeding mechanism is placed on top of the tank, it consist of a DC motor with screw conveyer that deliver the fish feed when the motor get actuated. An air pump is used to aerate the water, that is essential for fish life and it also help the decomposition of fish excretion and waste food.

Hydroponics part mainly consists of a water pump that used to pump nutrient and mineral rich water from the tank. The plants are placed on the pipe with help of supporting that help to hold the plant in vertical position. The one end of the pipe connects with the pump and other end return to tank. The filter attached to the pump inlet will filter out the solid particle to avoid blockage in the pipe. In order to ensure the continuity of water, a water detector is placed the end of the pipe. The water detector output can be used to alarm the operator or turn on a spare pump when the main pump fails. Butterfly valve in between the Pipe and pump ensure one direction flow of water.

The entire setup is placed in a greenhouse so that it is independent on the climate and climate changes. In certain climate particularly in monsoon season the sun light may not be sufficient to for the crops. So we need to provide artificial light to compensate the changes. The LED arrays with LDR are provided to keep the particular light level inside the greenhouse. White LED's are used such that their color can be changed using light filter of suitable wavelength. Pulse Width Modulation (PWM) is used to control the current flowing through the LED's for reducing energy loss in transistor.

Humidity plays an important role in greenhouse management. Humidity should be in a particular level depends on the characteristics of crop, its growth and yielding. Humidity sensor HRT 393 is used to monitor the humidity level in the green house. Temperature has great effect on humidity and if it is higher than the required level the crop get dehydrated. So temperature should be monitored continuously. For humidity control feed forward control strategy is used. Humidity is inversely proportional to temperature. In this case temperature is considered as the disturbance. So the controller parameter should be tuned properly to nullify the effect of temperature in humidity by activating humidifier. For the experimental purpose an air cooler is used. Humidifier motor starts working if the greenhouse temperature crosses the higher level and turn off if reaches in the prescribed level corresponding to required humidity. The farm operator may not be situated near to the greenhouse. So an online operating facility is implemented so the plant can be operated from anywhere if internet facility is available.

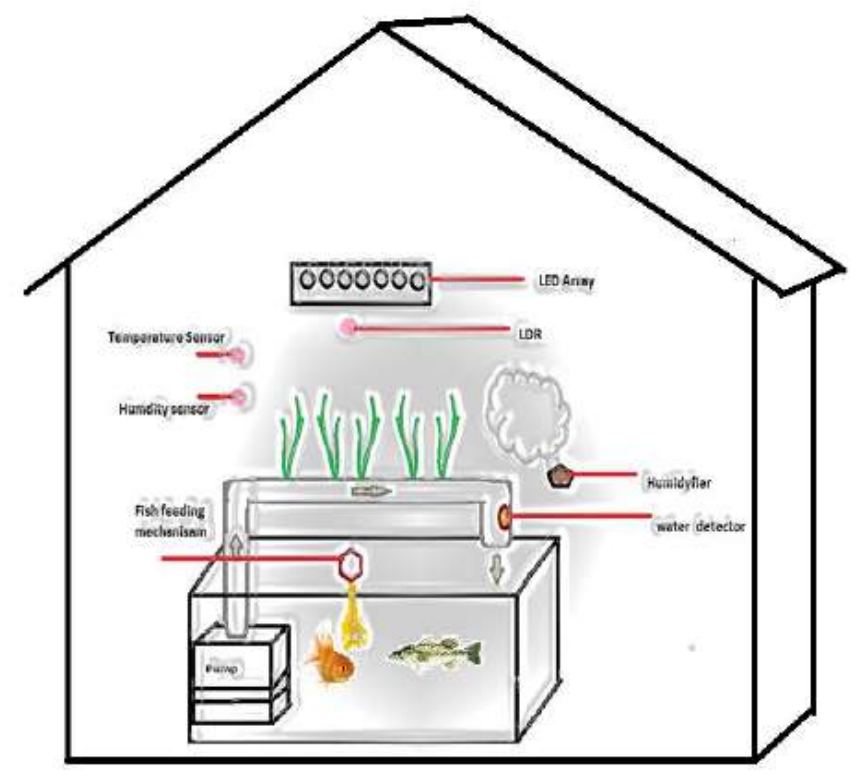

Fig 2 Aquaponics greenhouse setup

For accommodating these controls and monitoring strategies LabVIEW is installed in the server system and the client can be any PC or mobile with suitable plugin installed. To interface sensor and actuator Arduino-uno board is used with USB interface. The block diagram of the soilless farming is shown in Fig: 3. The LabVIEW software is installed in the main server having required hardware configuration. The server can access from any computer system if the networking facility is provided. Server can be accessed by typing the URL in any web browser. The operator can control the plant through the web browser after the server gives access control. In order acquire data from the sensor LabVIEW require data acquisition cards. But data acquisition card provided by the National Instruments are costly hence low cost Arduino board is preferred. Arduino can only handle voltage range from 0 to $5 \mathrm{~V}$, so interfacing circuits are necessary to convert the transducers output in to the acceptable range. Temperature and Humidity sensor can be directly interface with Arduino but LDR and water detector probe need interfacing circuits. Interfacing circuit consists of operational amplifier with desired gain. 


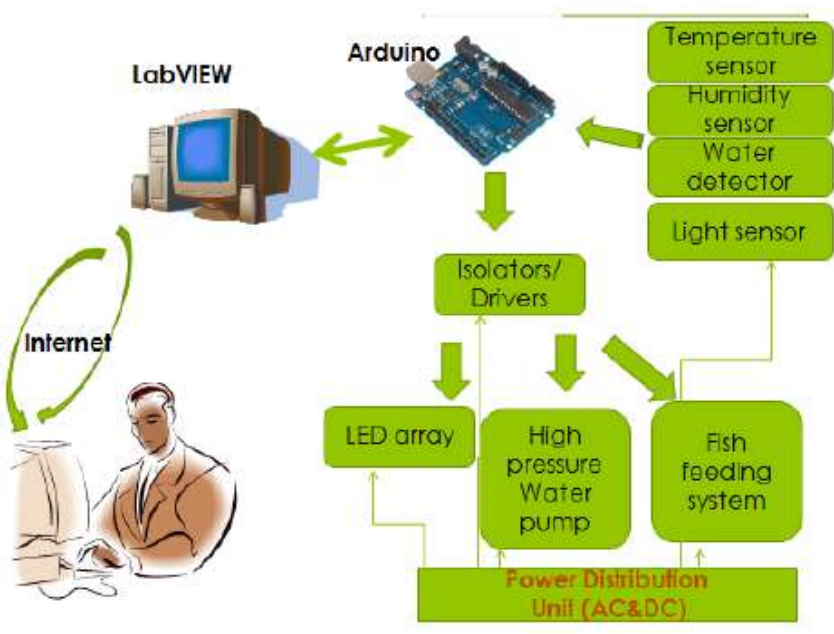

Fig 3 Block diagram of soilless farming

The Arduino output can be digital binary and PWM signal. LED and Fish Feed motors are rated at 12 DC and they are actuated using PWM signal by mean of SL100 transistor driven circuits. The Humidifier motor and water circulation pump are is rated at $230 \mathrm{AC}$, so relay are required for the operation. The solenoid relay require more current than that of the Arduino can provide, so transistors are used to amplify the signal.

\section{CONTROL STRATEGIES}

\subsection{Control Strategies for Light}

For lighting control a normal PID feedback control strategy is adequate. The required light output can be given by the front panel GUI of LabVIEW. The entered value is taken as the set point for the lighting control. With respect to the feedback given by the LDR the PID controller adjust the lighting by means of PWM. The driving circuit consisting SL100 transistor and biasing resistor has the role of actuator.

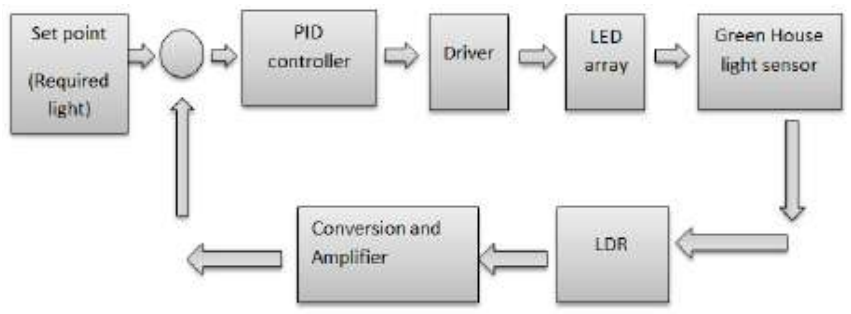

Fig 4 Control strategies for Light

\subsection{Control Strategy for Humidity}

For Humidity control feedback strategy can't be followed since, there is no meaning of controlling humidity when the crop get affected by dehydration. So a feed-forward strategy is followed here. Humidity decreases with increasing in temperature, so temperature can be taken as the disturbance to the system. The LM35 temperature sensor measures the temperature by considering as a disturbance to the humidity control system. The On-Off controller takes control action to keep the humidity level given by the operator to compensate the disturbance. There no chance of external humidified air entering inside the greenhouse so humidity doesn't rise above the limits.

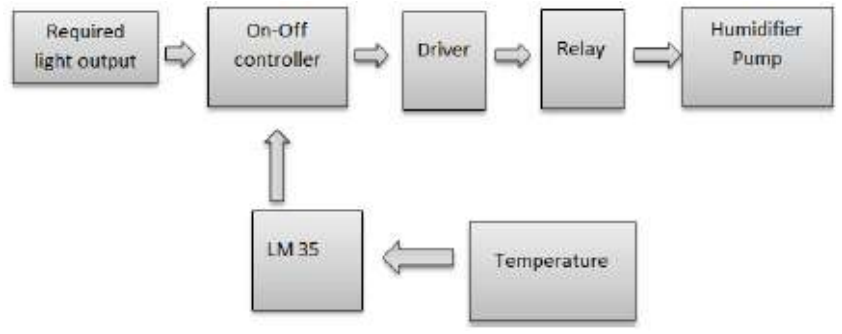

Fig 5 Control strategies for Humidity

\section{HARDWARE DESIGN}

\subsection{Circuit for Light Intensity Sensing}

Light sensing circuit consists of LDR, Operational amplifier and biasing resistors. LDR resistance decreases as increasing the intensity of light falling on the device. This resistance is converted to voltage by potential divider using a pot. This voltage is amplified using non-inverting Amplifier based on Op-amp circuit. Potentiometer is tuned to get proper voltage output in the range of 0 to $5 \mathrm{~V}$.

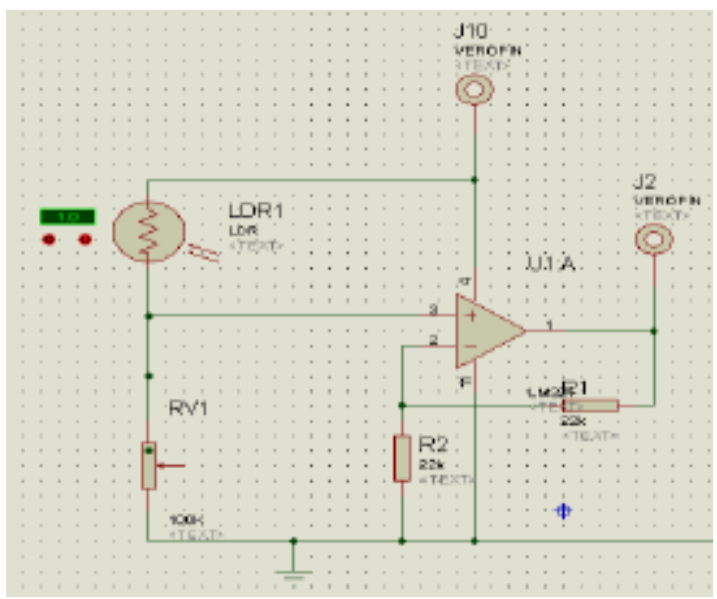

Fig 6 Circuits for light intensity detector

\subsection{Circuit for Water Continuity Detector}

Water continuity detector probe is nothing but a DC power jack pin with two contact terminals. The terminals produce low resistance when the tip is dipped in the mineralized water. The Operational amplifier is used as a comparator to detect the voltage change. The output will be low when water is circulating and high when water flow stops

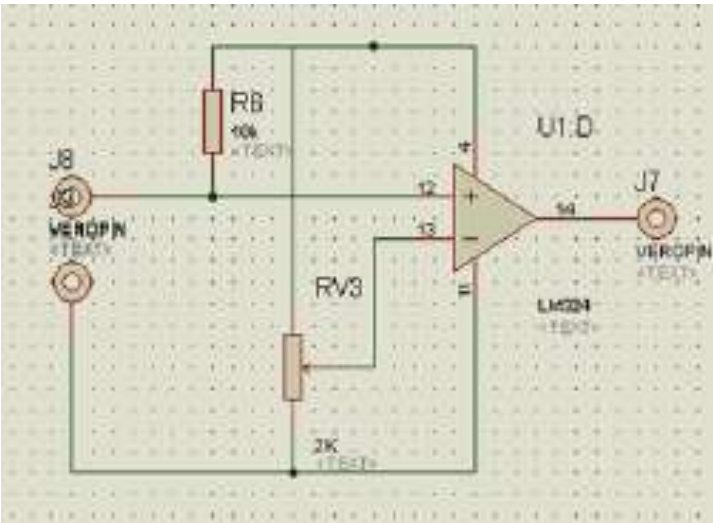

Fig 7 Circuits for water continuity detector 


\subsection{Driver Circuit}

The driver circuit consists of SL100 transistor and diode with proper biasing resistor. Transistor operates as a switch which turns on and off based on the voltage reaching at the base of transistor. Diode is used to block the reverse current flow when the transistor got damaged or faulty. This circuit is used for driving High Power LED's as well as 12V DC motor in the fish feeding mechanism. The motor drives the screw conveyer in the fish feeding mechanism.

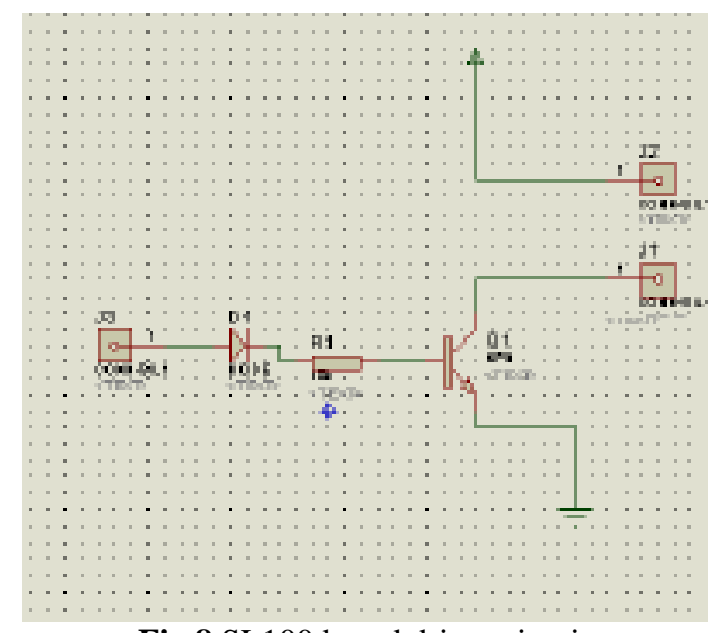

Fig 8 SL100 based driver circuit

\subsection{HRT 393 Humidity Sensor}

HRT 393 is a Relative Humidity (RH) sensor its conductivity varies when the adsorbed water condensed on the surface of the sensing material. The resistance is then converted voltage using proper circuit. The sensor gives the out in the range of 0 to $5 \mathrm{~V}$ when it is supplied with $5 \mathrm{~V} \mathrm{DC}$. The calibration is done inside the LabVIEW block diagram by adjusting the gain in the gain block. Humidity is representing in the relative humidity scale. Humidity measurement is using only for the monitoring purpose. Controlling action is triggered by temperature measurement by means of feed forward control.

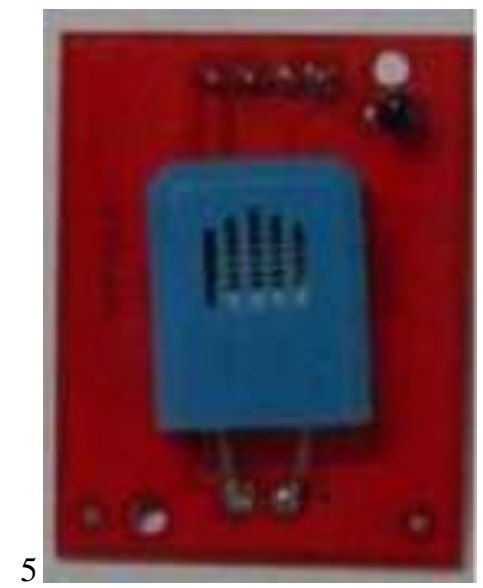

Fig 8 HRT 393

\subsection{Humidifier}

For the experimental purpose a water based air cooler is used as humidifier. The cooler takes dry air and mix with water droplets with help a fan. For large plant high pressure water pump are used.

\subsection{Fish Feeding Mechanisam}

The Fish Feeding Mechanism consists of a vessel with inverted cone at the bottom which is filled with fish food. The bottom cone connects with a screw conveyer and fitted on the top of fish tank. The shaft end is connected with a DC motor and the other end is free. As the motor rotates the shaft rotates and it pushes the fish food through the exist $t$. The screw teeth should have enough size to carry the fish food.

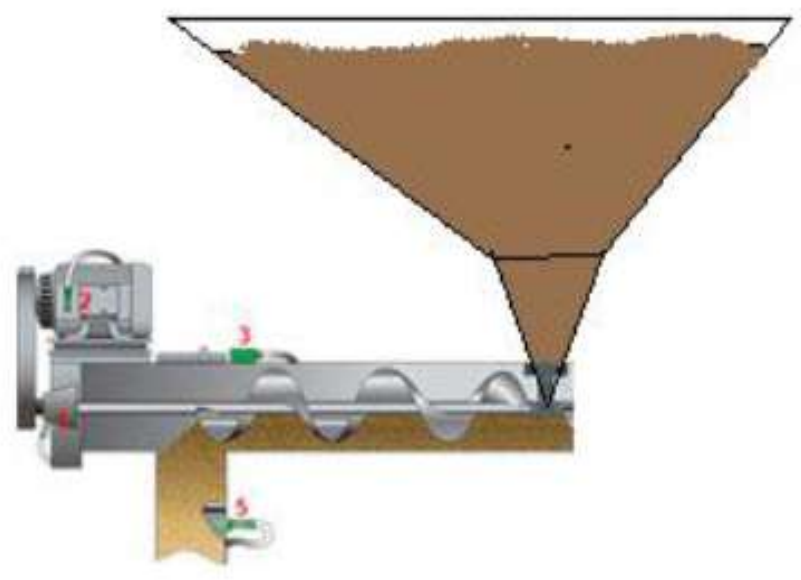

Fig 9 Screw conveyer based Fish Feeding Mechanism

\section{SOFTWARE DESIGNING}

Lab VIEW (Laboratory Virtual Instrument Engineering Workbench) is a system-design platform and development environment for a visual programming language from National Instruments. To interface Arduino boards with Lab VIEW installed systems, a software package "Live Interface for Arduino" is used.

\subsection{Front Panel}

Front panel consist of buttons, meter and text box for interfacing with the plant. The light intensity set point can be entered through the input box provided below the meter. It also display the error and controller output. By the controller strategy the humidifier pump start working whenever the temperature higher than the prescribed temperature. The humidifier working also visualized in the front panel. Water circulation is a crucial variable in the plant. If it fails the entire crop will be dehydrated. 


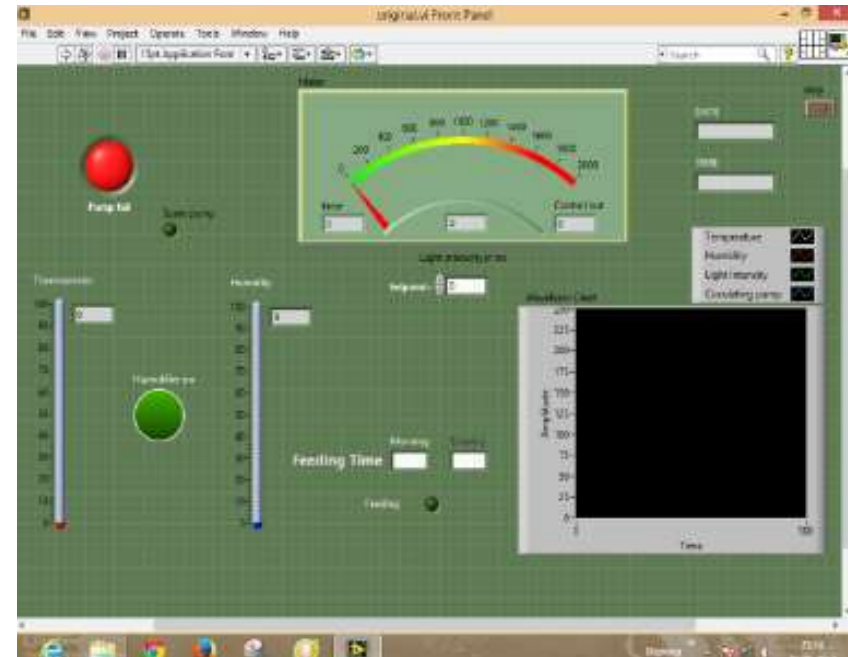

Fig 10 Front panel

The front panel indicates the circulating pump and it automatically trigger a spare pump whenever it fails. The fish feeding time can be entered through the front panel. The green LED indicates feeding. The entire signals associated with the system are represented in the form for chart for the easy of understand. Plant shutdown option also incorporated by means of a button for the maintenance work or if any emergency

\subsection{Block Diagram Design}

The block diagram connects the front panel control with the real signals by means of functions. The front panel components are connected to Arduino pins through suitable functional blocks. When the LabVIEW starts running it takes reading from the Arduino and the data coming from online to calculate the control signal. The control signal given out by means of Arduino itself. The signal is used to actuate motors, LED's and Humidifier by means of interfacing circuits.

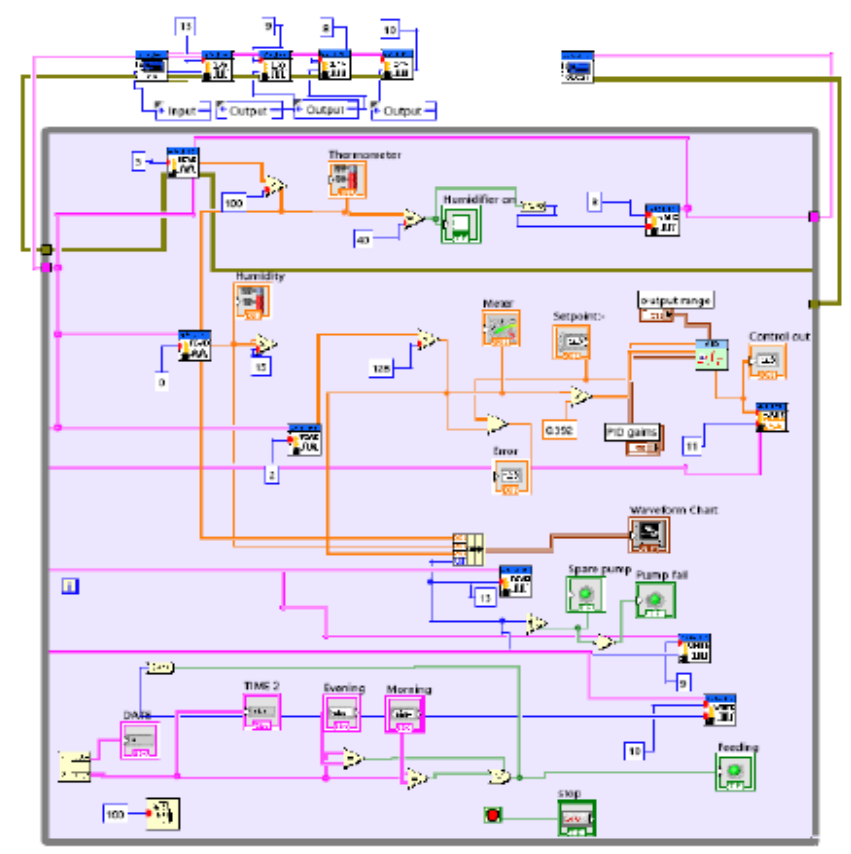

Fig 11 LabVIEW Block Diagram Design

\section{RESULTS AND DISCUSSION}

This designed system monitors the water circulation, temperature and the humidity greenhouse and takes control action to adjust the variation from the set point. The Fig 9.1 shows the result of light intensity control when the set point changes from 0 to 255 unit. The initial intensity in the green house is about 20 units.

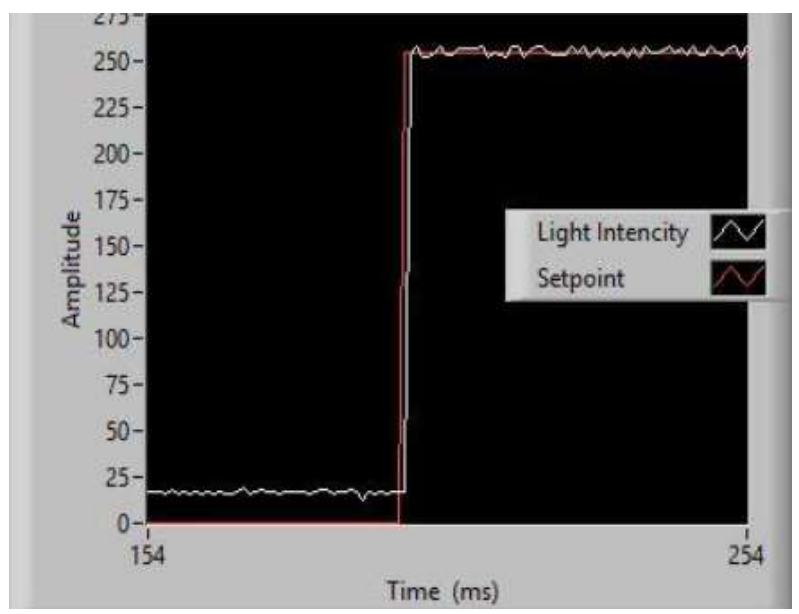

Fig 12 Light intensity tracking

The big red light on the front panel indicates pump failure. During pump failure current operating pump power supply cut off and an auxiliary pump get turned on to maintain the water flow. The fish feeding mechanism incorporated in the system can be configured through the front panel to feed the fish in the proper timing. The online monitor and control facility seem to be taking control actions by means of a webbrowser using server IP with port number.

\section{REFERENCES}

[1]. Francis Bacon, "Sylva Sylvarumby" Book of natural History 1627

[2]. Chang-Sun Shin, Yong-Woong Lee, Meong-Hun Lee, Jang-Woo Park, and Hyun Yoe, "Design of Ubiquitous Glass Green Houses",2009 Software Technologies for Future Dependable Distributed Systems IEEE transation by Computer Socity

[3]. Mitar Simic, "Design and Development of Air Temperature and Relative Humidity Monitoring System With AVR Processor Based Web Server" 2014 International Conference and Exposition on Electrical and Power Engineering (EPE 2014), 16-18 October, Iasi, Romania [4]. Thangavel Bhuvaneswari, Joshua Tan Hong Yao, "Automated Greenhouse," 2014 IEEE International Symposium on Robotics and Manufacturing Automation.

[5]. P. S. Asolkar, .Dr. U. S. Bhadade "An Effective Method of Controlling the Greenhouse and Crop Monitoring Using GSM" 2015 International Conference on Computing Communication Control and Automation 